\title{
Stochastic Geometry Analysis of Receiver Diversity in Cellular Networks with SWIPT
}

\author{
Tu Lam Thanh ${ }^{(1)}$, Marco Di Renzo ${ }^{(1)}$, and Justin P. Coon ${ }^{(2)}$ \\ (1) Laboratoire des Signaux et Systèmes, CNRS, CentraleSupélec, Université Paris Sud, Université Paris-Saclay, France \\ (2) Oxford University - Department of Engineering Science, UK \\ e-mail: \{lamthanh.tu, marco.direnzo\}@12s.centralesupelec.fr, justin.coon@eng.ox.ac.uk
}

\begin{abstract}
In this paper, we study the feasibility of receiver diversity for application to downlink cellular networks, where low-energy devices are equipped with information decoding and energy harvesting receivers for simultaneous wireless information and power transfer. We study several options that are based on selection combining and maximum ratio combining. Monte Carlo simulations are used to validate our findings and to discuss the trade-offs that emerge in cellular networks with simultaneous wireless information and power transfer.
\end{abstract}

\section{INTRODUCTION}

The Internet of Things (IoT) is expected to connect billions of Low-Energy Devices (LEDs) by 2020 [1]. One of the main challenges of the IoT is the provision of enough energy for the electronics of the LEDs, in order to have them operational over a reasonable amount of time and without making their battery too large or the device itself too bulky. In this context, the emerging concept of Simultaneous Wireless Information and Power Transfer (SWIPT) constitutes a suitable solution for prolonging the battery life of the LEDs and for making them energy-neutral, i.e., operational in a self-powered fashion.

The design of cellular networks with SWIPT introduces, however, new research challenges and optimization tradeoffs. Conventional cellular networks are designed based on the assumption that the interference has a negative impact on Information Decoding (ID), since it reduces the coverage and rate [2]. The same interference, on the other hand, is a natural source of power for Energy Harvesting (EH) [3]. In this context, receiver diversity turns out to be a promising solution for enhancing the reliability of data transmission and for increasing the amount of harvested power [4]. Receiver diversity is a practical solution to take advantage of multiple antenna elements by using few radio frequency chains.

The potential of receiver diversity for application to SWIPTenabled systems has recently been analyzed in [5] and [6]. In these papers, in particular, it has been shown that receiver diversity based on selection combining and antenna switching is a promising alternative compared with typical approaches based on power splitting and time switching. As elaborated in [6], power splitting and time switching need dedicated hardware components (power splitters and time switches), which may increase the complexity and cost of the LEDs and may be subject to efficiency losses. Time switching necessitates dedicated time slots and synchronization circuits for EH, which results in the discontinuous transmission of information data.
Receiver diversity, on the other hand, is a mature technology that may overcome these limitations. It requires, however, the availability of multiple antenna elements at the LEDs. This leads to new performance versus implementation complexity trade-offs that are not totally understood.

Motivated by these considerations, we study the potential of receiver diversity for application to SWIPT-enabled cellular networks. We focus our attention on practical implementations where one or two receive antennas are available at the LEDs. This implies that only one or two radio frequency chains are needed. More antenna elements may be used, by still employing one or two radio frequency chains, for LEDs of larger size, e.g., for relay nodes [4]. We study various options based on selection combining and maximum ratio combining schemes and focus on system-level analysis and optimization rather than on link-level optimization. More specifically, we take into account the impact of large-scale network deployments and introduce new analytical frameworks for the adaptive optimization of SWIPT-enabled cellular networks. This is performed by exploiting the mathematical tool of stochastic geometry and by modeling the locations of cellular Base Stations (BSs) as points of a Poisson Point Process (PPP). Compared with recent papers that have exploited similar mathematical tools for large-scale analysis of wireless networks with SWIPT, e.g., [7], our paper is focused on the potential of receiver diversity for application to cellular networks.

This paper is organized as follows. In Section II, the system model is introduced. In Section III, the research problem is first motivated and then formulated in terms of the Joint Complementary Cumulative Distribution Function (J-CCDF) of information rate and harvested power. In Section IV, analytical frameworks for system-level analysis are introduced. In Section V, analysis and findings are validated with the aid of numerical simulations. Section VI concludes this paper.

\section{System Model}

\section{A. Cellular Networks Modeling}

A downlink cellular network is considered. The BSs are modeled as points of a homogeneous PPP, denoted by $\Psi$, of density $\lambda$. The transmit power of the BSs is fixed and is denoted by $P$. Without loss of generality, the analysis is performed for the typical LED located at the origin [2]. 


\section{B. Channel Modeling}

The channel model accounts for Line-of-Sight (LOS) and Non-LOS (NLOS) links due to spatial blockages, for the pathloss, and for the fast-fading. Shadowing is implicitly taken into account via the LOS and NLOS link model [8].

1) LOS/NLOS Links: Let $r$ be the distance from a BS to the typical LED. The probability of LOS and NLOS as a function of $r, p_{s}(\cdot)$ for $s \in\{\mathrm{LOS}, \mathrm{NLOS}\}$, is formulated as follows:

$$
p_{s}(r)=\left\{\begin{array}{lll}
q_{s}^{[0, D]} & \text { if } & r \in[0, D) \\
q_{s}^{[D, \infty]} & \text { if } & r \in[D,+\infty)
\end{array}\right.
$$

where $q_{\mathrm{LOS}}^{[a, b]}+q_{\mathrm{NLOS}}^{[a, b]}=1,0 \leq q_{s}^{[a, b]} \leq 1$ is the probability that a link of length $r \in[a, b)$ is in state $s$, and $D$ takes into account that LOS and NLOS probabilities are different for short and long distances [8]. Assuming no spatial correlation among the links, $\Psi$ can be split in two independent and non-homogeneous PPPs, $\Psi_{\mathrm{LOS}}$ and $\Psi_{\mathrm{NLOS}}$, such that $\Psi=\Psi_{\mathrm{LOS}} \cup \Psi_{\mathrm{NLOS}}$. From (1) and the thinning theorem of PPPs, the density of $\Psi_{s}$ is $\lambda_{s}(r)=\lambda p_{s}(r)$ for $s \in\{\mathrm{LOS}, \mathrm{NLOS}\}$.

2) Path-Loss: The path-loss of LOS and NLOS links is $l_{s}(r)=\kappa_{0} r^{\beta_{s}}$ for $s \in\{\mathrm{LOS}, \mathrm{NLOS}\}$, where $\kappa_{0}=(4 \pi / \nu)^{2}$, $\nu$ is the transmission wavelength, $\beta_{s}$ is the path-loss exponent.

3) Fast-Fading: The channel gains are independent and identically distributed (i.i.d.) complex Gaussian Random Variables (RVs) with zero mean and unit variance, i.e., Rayleigh fading is considered.

\section{Cell Association}

The typical LED is served by the BS providing the smallest path-loss. The other BSs act as interferers. The smallest pathloss can be formulated as $L^{(0)}=\min \left\{L_{\mathrm{LOS}}^{(0)}, L_{\mathrm{NLOS}}^{(0)}\right\}$, where, for $s \in\{\operatorname{LOS}, \operatorname{NLOS}\}, L_{s}^{(0)}$ is the smallest path-loss of $\Psi_{s}$, which is defined as follows:

$$
L_{s}^{(0)}=\min _{n \in \Psi_{s}}\left\{l_{s}\left(r^{(n)}\right)\right\}
$$

where $r^{(n)}$ is the distance between the $n$th BS of $\Psi_{s}$ and the typical LED.

\section{Directional Beamforming at the BS}

At the BSs, to enhance the efficiency of information transmission and energy transfer over long distances, directional beamforming is used. Directional beamforming can be implemented by using, e.g., uniform linear arrays [7, Sec. II-C]. We consider a two-lobe model for the radiation pattern, where $\theta_{\mathrm{M}}$ is the beamwidth of the main lobe, and $G_{\mathrm{M}}$ and $G_{\mathrm{S}}$ are the beamforming gains of main and side lobes, respectively. The triplet $\left(\theta_{\mathrm{M}}, G_{\mathrm{M}}, G_{\mathrm{S}}\right)$ satisfies the unit power constraint, i.e., $\theta_{\mathrm{M}} G_{\mathrm{M}}+\left(2 \pi-\theta_{\mathrm{M}}\right) G_{\mathrm{S}}=2 \pi$. Due to their small size, the LEDs use omnidirectional antennas with a unit gain.

The typical LED and its serving BS estimate the angles of arrival and adjust their antenna steering orientations accordingly. Thus, the antenna gain of the typical intended link is $G^{(0)}=G_{\mathrm{M}}$. From the perspective of the typical LED, on the other hand, the beams of all interfering BSs are randomly oriented, i.i.d., and uniformly distributed in $[0,2 \pi)$. Thus, the Probability Density Function (PDF) of the antenna gain of the $i$ th interfering link, $G^{(i)}$, is:

$$
f_{G^{(i)}}(g)=\frac{\theta_{\mathrm{M}}}{2 \pi} \delta\left(g-G_{\mathrm{M}}\right)+\left(1-\frac{\theta_{\mathrm{M}}}{2 \pi}\right) \delta\left(g-G_{\mathrm{S}}\right)
$$

where $\delta(\cdot)$ is Dirac delta function.

\section{E. SWIPT and Receiver Diversity at the LEDs}

Due to their small form factor, the LEDs cannot accommodate many receive antennas. Hence, we analyze the case studies where the number of receive antennas and radio frequency chains, $N_{r}$, is either $N_{r}=1$ or $N_{r}=2$. These two setups find practical application to wrist-worn LEDs, e.g., smart watches, since the average circumference of a human wrist is about $14-20 \mathrm{~cm}$. The LEDs are equipped with separate units for ID and EH. To shed light on the impact of receiver diversity, five schemes for SWIPT are studied and compared.

- Power Splitting (PS). $N_{r}=1$ is assumed and the received power, $\mathrm{P}_{\mathrm{RX}}$, is split in two parts, according to a power splitting ratio $0 \leq \rho \leq 1: \mathrm{P}_{\mathrm{EH}}=\rho \mathrm{P}_{\mathrm{RX}}$ is used for $\mathrm{EH}$ and $\mathrm{P}_{\mathrm{ID}}=\mathrm{P}_{\mathrm{RX}}-\mathrm{P}_{\mathrm{EH}}=(1-\rho) \mathrm{P}_{\mathrm{RX}}$ is used for ID.

- Power Splitting with Maximum Ratio Combining (PSMRC). $N_{r}=2$ is assumed and the signals of the two receive antennas are combined according to the MRC scheme. The power after combining, $\mathrm{P}_{\mathrm{RX}}$, is split in two parts, according to a power splitting ratio $0 \leq \rho \leq 1$ : $\mathrm{P}_{\mathrm{EH}}=\rho \mathrm{P}_{\mathrm{RX}}$ is used for $\mathrm{EH}$ and $\mathrm{P}_{\mathrm{ID}}=\mathrm{P}_{\mathrm{RX}}-\mathrm{P}_{\mathrm{EH}}=$ $(1-\rho) \mathrm{P}_{\mathrm{RX}}$ is used for ID.

- Separate Antenna Receiver (SAR). $N_{r}=2$ is assumed and the received power of the first and second receive antenna is sent, without loss of generality, to the input of the ID and EH unit, respectively. The two antennas can be used for ID and EH interchangeably.

- ID-Prioritized Selection Combining (ID-SC). $N_{r}=2$ is assumed and the received power of the antenna providing the best and the worst channel power gain is sent to the input of the ID and $\mathrm{EH}$ unit, respectively.

- EH-Prioritized Selection Combining (EH-SC). $N_{r}=2$ is assumed and the received power of the antenna providing the best and the worst channel power gain is sent to the input of the $\mathrm{EH}$ and ID unit, respectively.

The proposed study can be generalized for application to SWIPT implementations based on the time switching scheme [7]. For brevity, this case study is not analyzed in this paper.

\section{Problem Statement}

Considered individually, the performance of ID and EH units is usually quantified in terms of information rate and harvested power, respectively. Let $\mathcal{R}$ and $\mathcal{Q}$ denote the achievable rate (in bit/sec) of the ID unit and the harvested power (in Watts) of the EH unit, respectively. As far as the five SWIPT schemes introduced in Section II-E are concerned, $\mathcal{R}$ and $\mathcal{Q}$ 
TABLE I

DEFINITION AND DISTRIBUTION OF $\mathcal{U}_{z}^{(0)}, \gamma_{z}^{(i)}$ AND $\rho_{z}$ IN (4) FOR THE FIVE SWIPT SCHEMES IN SECTION II-E. $\gamma_{r}^{(0)} \sim \mathcal{E}(1)$ AND $\gamma_{r}^{(i)} \sim \mathcal{E}(1)$ ARE THE CHANNEL POWER GAINS OF INTENDED AND $i$ TH INTERFERING BSS AT THE $r$ TH RECEIVE ANTENNA; $X \sim \mathcal{E}(\mu): X$ IS A RV WHOSE PDF IS

$f_{X}(\xi)=\mu \exp (-\mu \xi) ; X \sim \mathcal{E}_{\max }(1): X$ IS A RV WhOSE PDF IS $f_{X}(\xi)=2 \exp (-\xi)-2 \exp (-2 \xi) ; X \sim \mathcal{G}(2,1): X$ IS A RV WHOSE PDF IS $f_{X}(\xi)=\xi \exp (-\xi) ; \gamma_{\text {MRC }}^{(i)}, \gamma_{\max }^{(i)}$, AND $\gamma_{\min }^{(i)}$ ARE THE CHANNEL POWER GAINS OF THE $i$ TH INTERFERING BS AFTER APPLYING MRC AND IMPINGING ON THE BEST AND WORST (AS FAR AS THE THE PROBE LINK IS CONCERNED) RECEIVE ANTENNAS, RESPECTIVELY.

\begin{tabular}{|c|c|c|c|c|c|c|c|c|}
\hline & \multicolumn{2}{|l|}{$\mathcal{U}_{\mathrm{ID}}^{(0)}$} & \multicolumn{2}{|l|}{$\mathcal{U}_{\mathrm{EH}}^{(0)}$} & $\gamma_{\mathrm{ID}}^{(i)}$ & $\gamma_{\mathrm{EH}}^{(i)}$ & $\rho_{\text {ID }}$ & $\rho_{\mathrm{EH}}$ \\
\hline PS & \multicolumn{2}{|c|}{$\gamma_{1}^{(0)} \sim \mathcal{E}(1)$} & \multicolumn{2}{|c|}{$\gamma_{1}^{(0)} \sim \mathcal{E}(1)$} & $\gamma_{1}^{(i)} \sim \mathcal{E}(1)$ & $\gamma_{1}^{(i)} \sim \mathcal{E}(1)$ & $1-\rho$ & $\rho$ \\
\hline PS-MRC & \multicolumn{2}{|c|}{$\gamma_{1}^{(0)}+\gamma_{2}^{(0)} \sim \mathcal{G}(2,1)$} & \multicolumn{2}{|c|}{$\gamma_{1}^{(0)}+\gamma_{2}^{(0)} \sim \mathcal{G}(2,1)$} & $\gamma_{\mathrm{MRC}}^{(i)} \sim \mathcal{E}(1)$ & $\gamma_{\mathrm{MRC}}^{(i)} \sim \mathcal{E}(1)$ & $1-\rho$ & $\rho$ \\
\hline SAR & \multicolumn{2}{|c|}{$\gamma_{1}^{(0)} \sim \mathcal{E}(1)$} & \multicolumn{2}{|c|}{$\gamma_{2}^{(0)} \sim \mathcal{E}(1)$} & $\gamma_{1}^{(i)} \sim \mathcal{E}(1)$ & $\gamma_{2}^{(i)} \sim \mathcal{E}(1)$ & 1 & 1 \\
\hline ID-SC & $\max \left\{\gamma_{1}^{(0)}, \gamma_{2}^{(0)}\right\}$ & $\sim \mathcal{E}_{\max }(1)$ & $\min \left\{\gamma_{1}^{(0)}, \gamma_{2}^{(0)}\right\}$ & $\sim \mathcal{E}(2)$ & $\gamma_{\max }^{(i)} \sim \mathcal{E}(1)$ & $\gamma_{\min }^{(i)} \sim \mathcal{E}(1)$ & 1 & 1 \\
\hline EH-SC & $\min \left\{\gamma_{1}^{(0)}, \gamma_{2}^{(0)}\right.$ & $\sim \mathcal{E}(2)$ & $\max \left\{\gamma_{1}^{(0)}, \gamma_{2}^{(0)}\right\}$ & $\sim \mathcal{E}_{\max }(1)$ & $\gamma_{\min }^{(i)} \sim \mathcal{E}(1)$ & $\gamma_{\max }^{(i)} \sim \mathcal{E}(1)$ & 1 & 1 \\
\hline
\end{tabular}

can be formulated as follows:

$$
\begin{aligned}
& \mathcal{R}=B_{w} \log _{2}\left(1+\frac{P G^{(0)} \mathcal{U}_{\mathrm{ID}}^{(0)} / L^{(0)}}{P \mathcal{I}_{\mathrm{ID}}\left(L^{(0)}\right)+\sigma_{\mathrm{N}}^{2}+\sigma_{\mathrm{ID}}^{2} / \rho_{\mathrm{ID}}}\right) \\
& \mathcal{Q}=\rho_{\mathrm{EH}} \zeta\left(P G^{(0)} \mathcal{U}_{\mathrm{EH}}^{(0)} / L^{(0)}+P \mathcal{I}_{\mathrm{EH}}\left(L^{(0)}\right)\right)
\end{aligned}
$$

where $B_{w}$ is the transmission bandwidth and $\sigma_{\mathrm{N}}^{2}, \sigma_{\mathrm{ID}}^{2}$ are the thermal noise power and noise power due to the signal conversion from radio frequency to baseband, respectively, $0 \leq \zeta \leq 1$ is the efficiency of energy harvesting conversion, $0 \leq \rho_{z} \leq 1$, for $z \in\{\mathrm{ID}, \mathrm{EH}\}$, accounts for the amount of power at the input of ID and $\mathrm{EH}$ units, $\mathcal{U}_{z}^{(0)}$ is the power gain of the intended link, and $\mathcal{I}_{z}(\cdot)$ is the aggregate other-cell interference defined as follows:

$\mathcal{I}_{z}\left(L^{(0)}\right)=\sum_{s \in\{\mathrm{LOS}, \mathrm{NLOS}\}} \sum_{i \in \Psi_{s}} \frac{G^{(i)} \gamma_{z}^{(i)}}{l_{s}\left(r^{(i)}\right)} \mathbf{1}\left(l_{s}\left(r^{(i)}\right)>L^{(0)}\right)$

where $\gamma_{z}^{(i)}$ is the power gain of the $i$ th interfering BS and $\mathbf{1}(\cdot)$ is the indicator function. The definitions and the distributions of the parameters $\mathcal{U}_{z}^{(0)}, \gamma_{z}^{(i)}$ and $\rho_{z}$ are given in Table I.

Remark 1: Based on Table I, $\mathcal{I}_{\mathrm{ID}}(\cdot)=\mathcal{I}_{\mathrm{EH}}(\cdot)$ for PS and PS-MRC schemes, but $\mathcal{I}_{\text {ID }}(\cdot) \neq \mathcal{I}_{\mathrm{EH}}(\cdot)$ for SAR, ID-SC and EH-SC schemes. As far as the latter three SWIPT schemes are concerned, however, $\mathcal{I}_{\mathrm{ID}}(\cdot) \stackrel{d}{=} \mathcal{I}_{\mathrm{EH}}(\cdot)^{1}$. More precisely, $\mathcal{I}_{\mathrm{ID}}(\cdot)$ and $\mathcal{I}_{\mathrm{EH}}(\cdot)$ are partially correlated RVs because the locations of the interfering BSs are the same but the power channel gains are related to different receive antennas. For all SWIPT schemes, the distribution of the aggregate other-cell interference is the same, i.e., $\Phi_{\mathcal{I}_{\mathrm{ID}}}(\cdot)=\Phi_{\mathcal{I}_{\mathrm{EH}}}(\cdot)=\Phi_{\mathcal{I}}(\cdot)$.

As far as the LEDs as a whole are concerned, the trade-off between information rate and harvested power is quantified in terms of the J-CCDF of $\mathcal{R}$ and $\mathcal{Q}$ defined in (4) as [7]:

$$
\bar{F}\left(\mathcal{R}_{*}, \mathcal{Q}_{*}\right)=\operatorname{Pr}\left\{\mathcal{R} \geq \mathcal{R}_{*}, \mathcal{Q} \geq \mathcal{Q}_{*}\right\}
$$

where $\mathcal{R}_{*}$ and $\mathcal{Q}_{*}$ are the minimum bit rate and harvested

\footnotetext{
${ }^{1} X \stackrel{d}{=} Y$ : RVs $X, Y$ are equivalent in distribution, i.e., their Characteristic Functions (CFs) and Moment Generating Functions (MGFs) are the same.
}

power, respectively, needed for the LEDs to perform their tasks, and $\operatorname{Pr}\{\cdot\}$ is the probability operator.

\section{SySTEM-LEVEL ANALYSIS}

The following two propositions provide computable expressions of the J-CCDF for the SWIPT schemes introduced in Section II-E. Proposition 1 is exact and is applicable only to PS and PS-MRC schemes. Proposition 2 is based on the Frechet upper-bound defined as follows [9]:

$$
\begin{aligned}
\bar{F}\left(\mathcal{R}_{*}, \mathcal{Q}_{*}\right) & \leq \bar{F}_{\mathrm{UB}}\left(\mathcal{R}_{*}, \mathcal{Q}_{*}\right) \\
& =\min \left\{\operatorname{Pr}\left\{\mathcal{R} \geq \mathcal{R}_{*}\right\}, \operatorname{Pr}\left\{\mathcal{Q} \geq \mathcal{Q}_{*}\right\}\right\}
\end{aligned}
$$

and is applicable to all SWIPT schemes.

Exact analytical frameworks for SAR, ID-SC and EH-SC schemes may be obtained by using the multi-dimensional inversion theorem. The mathematical complexity of studying SAR, ID-SC and EH-SC schemes compared with PS and PS-MRC schemes originates from the fact that the aggregate other-cell interferences of ID and EH units are dissimilar and are only equivalent in distribution (Remark 1).

Proposition 1: Let $f_{L^{(0)}}(\cdot)$ be the PDF of the smallest pathloss, $L^{(0)}$, defined in (2) and given as follows:

$$
f_{L^{(0)}}(x)=\widehat{\Lambda}([0, x)) \exp (-\Lambda([0, x)))
$$

where $\Lambda([0, x))=\Lambda_{\mathrm{LOS}}([0, x))+\Lambda_{\mathrm{NLOS}}([0, x))$ is the intensity measure of the PPP of the path-losses [7], $\widehat{\Lambda}([0, x))=$ $\widehat{\Lambda}_{\mathrm{LOS}}([0, x))+\widehat{\Lambda}_{\mathrm{NLOS}}([0, x))$ is its first derivative computed with respect to $x$, and, for $s \in\{\operatorname{LOS}, \operatorname{NLOS}\}, \Lambda_{s}([\cdot, \cdot))$ and $\widehat{\Lambda}_{s}([\cdot, \cdot))$ are the intensity measure and its first derivative, respectively, of the PPP of the path-losses in state $s$ :

$$
\begin{aligned}
& \Lambda_{s}([0, x)) \\
& =\pi \lambda q_{s}^{[0, D]}\left(\frac{x}{\kappa_{0}}\right)^{\frac{2}{\beta_{s}}} \overline{\mathcal{H}}\left(x-\kappa_{0} D^{\beta_{s}}\right)+\mathcal{H}\left(x-\kappa_{0} D^{\beta_{s}}\right) \\
& \times \pi \lambda\left(\left(\frac{x}{\kappa_{0}}\right)^{\frac{2}{\beta_{s}}} q_{s}^{[D, \infty]}+D^{2}\left(q_{s}^{[0, D]}-q_{s}^{[D, \infty]}\right)\right)
\end{aligned}
$$




$$
\begin{aligned}
& \Phi_{\mathcal{I}}\left(\omega \mid L^{(0)} ; s\right)=\exp \left(\lambda \pi q_{s}^{[D, \infty]} \max \left\{D^{2},\left(L^{(0)} / \kappa_{0}\right)^{2 / \beta_{s}}\right\}\left(1-\Upsilon_{s}\left(\omega, \max \left\{\kappa_{0} D^{\beta_{s}}, L^{(0)}\right\}\right)\right)\right) \\
& \quad \times \exp \left(\pi \lambda q_{s}^{[0, D]}\left[\left(L^{(0)} / \kappa_{0}\right)^{2 / \beta_{s}}\left(1-\Upsilon_{s}\left(\omega, L^{(0)}\right)\right)-D^{2}\left(1-\Upsilon_{s}\left(\omega, \kappa_{0} D^{\beta_{s}}\right)\right)\right] \overline{\mathcal{H}}\left(L^{(0)}-\kappa_{0} D^{\beta_{s}}\right)\right)
\end{aligned}
$$

$$
\begin{aligned}
& \widehat{\Lambda}_{s}([0, x)) \\
& =\left(2 \pi \lambda / \beta_{s}\right) q_{s}^{[0, D]} \kappa_{0}^{-2 / \beta_{s}} x^{\left(2 / \beta_{s}-1\right)} \overline{\mathcal{H}}\left(x-\kappa_{0} D^{\beta_{s}}\right) \\
& +\left(2 \pi \lambda / \beta_{s}\right) q_{s}^{[D, \infty]} \kappa_{0}^{-2 / \beta_{s}} x^{\left(2 / \beta_{s}-1\right)} \mathcal{H}\left(x-\kappa_{0} D^{\beta_{s}}\right)
\end{aligned}
$$

where $\mathcal{H}(\cdot), \overline{\mathcal{H}}(x)=1-\mathcal{H}(x)$, are the the Heaviside and complementary Heaviside function, respectively.

Let $\Phi_{\mathcal{I}}\left(\cdot \mid L^{(0)}\right)$ be the CF of the other-cell interference, $\mathcal{I}$, conditioned on $L^{(0)}$ given in (5):

$$
\Phi_{\mathcal{I}}\left(\omega \mid L^{(0)}\right)=\Phi_{\mathcal{I}}\left(\omega \mid L^{(0)} ; \mathrm{LOS}\right) \Phi_{\mathcal{I}}\left(\omega \mid L^{(0)} ; \mathrm{NLOS}\right)
$$

where, for $s \in\{\mathrm{LOS}, \mathrm{NLOS}\}, \Phi_{\mathcal{I}}\left(\cdot \mid L^{(0)} ; s\right)$ is, conditioned on $L^{(0)}$, the $\mathrm{CF}$ of the other-cell interference of all links in state $s$ defined in (14) shown at the top of this page, and $\Upsilon_{s}(\cdot, \cdot)$ is the following short-hand function:

$$
\begin{aligned}
\Upsilon_{s}(\omega, \mathrm{Z}) & =\frac{\theta_{\mathrm{M}}}{2 \pi}{ }_{2} F_{1}\left(1,-\frac{2}{\beta_{s}}, 1-\frac{2}{\beta_{s}}, \frac{j \omega}{Z} G_{\mathrm{M}}\right) \\
& +\left(1-\frac{\theta_{\mathrm{M}}}{2 \pi}\right){ }_{2} F_{1}\left(1,-\frac{2}{\beta_{s}}, 1-\frac{2}{\beta_{s}}, \frac{j \omega}{Z} G_{\mathrm{S}}\right)
\end{aligned}
$$

where $j=\sqrt{-1}$ is the imaginary unit and ${ }_{2} F_{1}(\cdot, \cdot ; \cdot ; \cdot)$ is Gaussian hypergeometric function.

Then, the J-CCDF of both PS and PS-MRC schemes is:

$$
\begin{aligned}
& \bar{F}\left(\mathcal{R}_{*}, \mathcal{Q}_{*}\right) \\
& =\int_{0}^{+\infty} \int_{0}^{+\infty} \frac{1}{\pi \omega} \operatorname{Im}\left\{\mathcal{J}(\omega, y) \Phi_{\mathcal{I}}\left(\frac{\omega}{G^{(0)}} \mid y\right)\right\} f_{L^{(0)}}(y) d \omega d y
\end{aligned}
$$

where $\operatorname{Im}\{\cdot\}$ is the imaginary part operator, the following short-hand function is introduced:

$$
\begin{aligned}
\mathcal{J}(\omega, y) & =\exp \left(-j \omega \frac{q_{*}}{P G^{(0)}}\right)\left(1-\frac{j \omega}{y}\right)^{-(1+u)} \\
& \times \Gamma\left(1+u, \frac{\mathcal{T}_{*}}{P G^{(0)}}(y-j \omega)\right) \\
& -\exp \left(j \omega \frac{\sigma_{*}^{2}}{P G^{(0)}}\right)\left(1+\frac{j \omega r_{*}}{y}\right)^{-(1+u)} \\
& \times \Gamma\left(1+u, \frac{\mathcal{T}_{*}}{P G^{(0)}}\left(y+j \omega r_{*}\right)\right)
\end{aligned}
$$

and $u=0$ for PS and $u=1$ for PS-MRC schemes, respectively, $r_{*}=\left(2^{\mathcal{R}_{*} / B_{w}}-1\right)^{-1}, \sigma_{*}^{2}=\sigma_{N}^{2}+\sigma_{\mathrm{ID}}^{2}(1-\rho)^{-1}$; $q_{*}=\mathcal{Q}_{*}(\rho \zeta)^{-1}, \mathcal{T}_{*}=\left(q_{*}+\sigma_{*}^{2}\right) /\left(r_{*}+1\right)$, and $\Gamma(\cdot, \cdot)$ if the upper-incomplete gamma function.

Proposition 2: Let $f_{L^{(0)}}(\cdot)$ be the PDF in (10) and $\Phi_{\mathcal{I}}(\cdot \mid \cdot)$ be the $\mathrm{CF}$ in (13). Let $\widehat{\mathcal{M}}_{\mathcal{I}}(z)=d \mathcal{M}_{\mathcal{I}}(z) / d z$ be the

\section{TABLE II}

DEFINITIONS OF $\left(a_{\mathrm{ID}}, b_{\mathrm{ID}}, c_{\mathrm{ID}}\right)$ AND $\left(a_{\mathrm{EH}}, b_{\mathrm{EH}}, c_{\mathrm{EH}}\right)$ ACCORDING TO THE PDFS IN TABLE I.

\begin{tabular}{|c||c|c|c||c|c|c|}
\hline & $a_{\mathrm{ID}}$ & $b_{\mathrm{ID}}$ & $c_{\mathrm{ID}}$ & $a_{\mathrm{EH}}$ & $b_{\mathrm{EH}}$ & $c_{\mathrm{EH}}$ \\
\hline \hline PS & 1 & 0 & 0 & 1 & 0 & 0 \\
\hline PS-MRC & 0 & 0 & 1 & 0 & 0 & 1 \\
\hline SAR & 1 & 0 & 0 & 1 & 0 & 0 \\
\hline ID-SC & 2 & -2 & 0 & 0 & 2 & 0 \\
\hline EH-SC & 0 & 2 & 0 & 2 & -2 & 0 \\
\hline
\end{tabular}

first derivative of the MGF of the aggregate other-cell interference, i.e., $\mathcal{M}_{\mathcal{I}}(z)=\Phi_{\mathcal{I}}(-j z \mid z)$. Then, the JCCDF of the SWIPT schemes in Table I can be upperbounded as $\bar{F}\left(\mathcal{R}_{*}, \mathcal{Q}_{*}\right) \leq \min \left\{\bar{F}_{\mathrm{ID}}\left(\mathcal{R}_{*}\right), \bar{F}_{\mathrm{EH}}\left(\mathcal{Q}_{*}\right)\right\}$, where $\bar{F}_{\mathrm{ID}}\left(\mathcal{R}_{*}\right)=\operatorname{Pr}\left\{\mathcal{R} \geq \mathcal{R}_{*}\right\}$ and $\bar{F}_{\mathrm{EH}}\left(\mathcal{Q}_{*}\right)=$ $\operatorname{Pr}\left\{\mathcal{Q} \geq \mathcal{Q}_{*}\right\}$ can be formulated, respectively, as follows:

$$
\begin{gathered}
\bar{F}_{\mathrm{ID}}\left(\mathcal{R}_{*}\right)=\int_{0}^{+\infty} \mathcal{J}_{\mathrm{ID}}(y) f_{L^{(0)}}(y) d y \\
\bar{F}_{\mathrm{EH}}\left(\mathcal{Q}_{*}\right)=\frac{1}{2}+\int_{0}^{+\infty} \int_{0}^{+\infty} \frac{1}{\pi \omega} \operatorname{Im}\left\{\exp \left(-j \omega \frac{\tilde{q}_{*}}{P}\right)\right. \\
\left.\times \mathcal{J}_{\mathrm{EH}}\left(\omega, \frac{G^{(0)}}{y}\right) \Phi_{\mathcal{I}}(\omega \mid y)\right\} f_{L^{(0)}}(y) d \omega d y
\end{gathered}
$$

where the following short-hand functions are introduced:

$$
\begin{aligned}
\mathcal{J}_{\mathrm{ID}}(y) & =\left(a_{\mathrm{ID}}+c_{\mathrm{ID}}\right) \exp \left(-\frac{\tilde{\sigma}_{*}^{2} y}{P G^{(0)} \tilde{r}_{*}}\right) \mathcal{M}_{\mathcal{I}}\left(\frac{y}{G^{(0)} \tilde{r}_{*}}\right) \\
& +\frac{b_{\mathrm{ID}}}{2} \exp \left(-\frac{2 \tilde{\sigma}_{*}^{2} y}{P G^{(0)} \tilde{r}_{*}}\right) \mathcal{M}_{\mathcal{I}}\left(\frac{2 y}{G^{(0)} \tilde{r}_{*}}\right) \\
& +c_{\mathrm{ID}} \frac{\tilde{\sigma}_{*}^{2} y}{P G^{(0)} \tilde{r}_{*}} \exp \left(-\frac{\tilde{\sigma}_{*}^{2} y}{P G^{(0)} \tilde{r}_{*}}\right) \mathcal{M}_{\mathcal{I}}\left(\frac{y}{G^{(0)} \tilde{r}_{*}}\right) \\
& -c_{\mathrm{ID}} \frac{y}{G^{(0)} \tilde{r}_{*}} \exp \left(-\frac{\tilde{\sigma}_{*}^{2} y}{P G^{(0)} \tilde{r}_{*}}\right) \widehat{\mathcal{M}}_{\mathcal{I}}\left(\frac{y}{G^{(0)} \tilde{r}_{*}}\right) \\
\mathcal{J}_{\mathrm{EH}}(\omega, z) & =a_{\mathrm{EH}}(1-j \omega z)^{-1}+b_{\mathrm{EH}}(2-j \omega z)^{-1} \\
& +c_{\mathrm{EH}}(1-j \omega z)^{-2}
\end{aligned}
$$

and the two triplets of coefficients $\left(a_{\mathrm{ID}}, b_{\mathrm{ID}}, c_{\mathrm{ID}}\right)$ and $\left(a_{\mathrm{EH}}, b_{\mathrm{EH}}, c_{\mathrm{EH}}\right)$ are defined in Table II; $\tilde{q}_{*}=\mathcal{Q}_{*}\left(\rho_{\mathrm{EH}} \zeta\right)^{-1}$; $\tilde{r}_{*}=r_{*}$ and $\tilde{\sigma}_{*}^{2}=\sigma_{N}^{2}+\sigma_{\mathrm{ID}}^{2} / \rho_{\mathrm{ID}}$.

The J-CCDFs in Proposition 1 and Proposition 2 are formulated in terms of two-fold integrals that can be efficiently computed with the aid of state-of-the-art computational software programs and have the advantage of avoiding lengthly Monte Carlo simulations. For brevity, the explicit expression of the first derivative of the MGF of the aggregate other-cell 


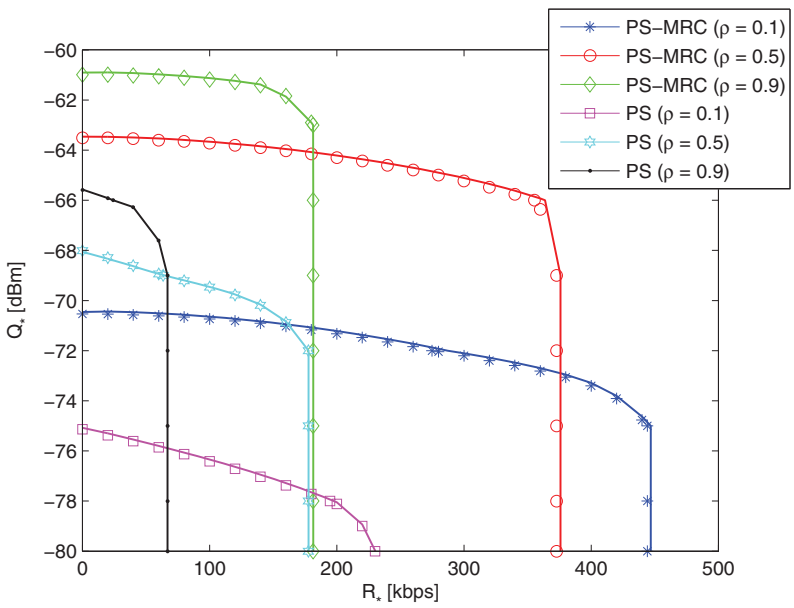

Fig. 1. Contour lines of the J-CCDF of PS and PS-MRC schemes as a function of $\rho$. The curves show the pairs $\left(\mathcal{R}_{*}, \mathcal{Q}_{*}\right)$ so that $\bar{F}\left(\mathcal{R}_{*}, \mathcal{Q}_{*}\right)=$ 0.75. Markers: Monte Carlo simulations. Solid lines: Proposition 1.

interference is not reported. It can be computed and formulated in closed-form from (13) and (14).

\section{Numerical AND Simulation RESUlts}

In this section, we use Monte Carlo simulations to validate our findings. Monte Carlo simulations are obtained as detailed in [8]. Unless otherwise stated, the following setup is considered: $\nu=c_{0} / f_{c}$, where $c_{0}$ is the speed of light in $\mathrm{m} / \mathrm{sec}$ and $f_{c}=2.1 \mathrm{GHz}$ is the carrier frequency; $\sigma_{\mathrm{ID}}^{2}=-70 \mathrm{dBm}$; $\sigma_{N}^{2}=-174+10 \log _{10}\left(B_{w}\right)+\mathcal{F}_{N} \mathrm{dBm}$, where $B_{w}=200$ $\mathrm{kHz}$ and $\mathcal{F}_{N}=10 \mathrm{~dB}$ is the noise figure; $P=30 \mathrm{dBm}$; $\zeta=0.8 ; \theta_{\mathrm{M}}=25.6$ degrees; $G_{\mathrm{M}}=7.47$; and $G_{\mathrm{S}}=0.5$. The channel model and the density of BSs, $\lambda$, are chosen in agreement with [8]: $D=109.8517 \mathrm{~m}, q_{\mathrm{LOS}}^{[0, D]}=0.7195$, $q_{\mathrm{LOS}}^{[D, \infty]}=0.0002, \beta_{\mathrm{LOS}}=2.5, \beta_{\mathrm{NLOS}}=3.5, \lambda=1 /\left(\pi R_{\text {cell }}^{2}\right)$ where $R_{\text {cell }}=83.4122 \mathrm{~m}$ is the average cell radius. In Figs. $1-2$, without loss of generality, we analyze the case study $\bar{F}\left(\mathcal{R}_{*}, \mathcal{Q}_{*}\right)=0.75$. Imposing higher values of the J-CCDF results in lower values of $\mathcal{R}_{*}$ and $\mathcal{Q}_{*}$ that satisfy them.

In Figs. 1 and 2, we validate the correctness of Proposition 1 and Proposition 2, respectively, against Monte Carlo simulations. In particular, Fig. 1 confirms that the J-CCDF in Proposition 1 is exact and Fig. 2 highlights that the Frechet inequality in Proposition 2 provides a upper-bound of the JCCDF, which is asymptotically tight as the system operates either in the ID-limited or in the EH-limited regimes. From the engineering standpoint, Figs. 1 and 2 show that information rate and harvested power highly depend on the choice of $\rho$ for PS and PS-MRC schemes, as well as that there is no scheme among SAR, ID-SC and EH-SC that outperform all the others for every pair $\left(\mathcal{R}_{*}, \mathcal{Q}_{*}\right)$.

\section{CONCLUSION}

In this paper, we have analyzed SWIPT-enabled cellular networks that employ several receiver diversity schemes. We have shown that receiver diversity has the potential of enhancing the information rate and of increasing the harvested power

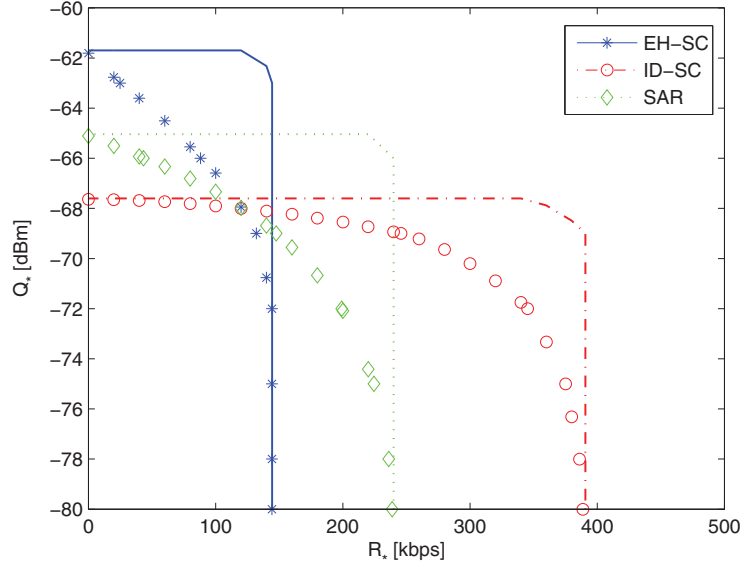

Fig. 2. Contour lines of the J-CCDF of SAR, ID-SC and EH-SC schemes The curves show the pairs $\left(\mathcal{R}_{*}, \mathcal{Q}_{*}\right)$ so that $\bar{F}\left(\mathcal{R}_{*}, \mathcal{Q}_{*}\right)=0.75$. Markers: Monte Carlo simulations. Solid, dotted and dashed lines: Proposition 2.

simultaneously. We have found, in addition, that the systemlevel performance can be improved by adaptively choosing the receiver diversity scheme as a function of the information rate and harvested power requirements that need to be fulfilled. An extended version of the present paper can be found in [10].

\section{ACKNOWLEDGMENT}

This work was supported in part by the European Commission through the H2020-MSCA ETN-5Gwireless project (grant 641985), the Agence Nationale de la Recherche Scientifique (ANR) through the research project SpatialModulation (Action Plan 2015), and the EPSRC through the Spatially Embedded Networks project (grant EP/N002350/1).

\section{REFERENCES}

[1] A. Al-Fuqaha et al., "Internet of things: A survey on enabling technologies, protocols, and applications", IEEE Commun. Surveys \& Tuts., vol. 17 , no. 4, pp. 2347-2376, 2015.

[2] M. Di Renzo et al., "Average rate of downlink heterogeneous cellular networks over generalized fading channels - A stochastic geometry approach", IEEE Trans. Commun., vol. 61, pp. 3050-3071, July 2013.

[3] G. Zheng et al., "Rethinking the role of interference in wireless networks", IEEE Commun. Mag., vol. 52, pp. 152-158, Nov. 2014.

[4] Z. Ding et al., "Application of smart antenna technologies in simultaneous wireless information and power transfer", IEEE Commun. Mag., vol. 53, no. 4, pp. 86-93, Apr. 2015.

[5] L. Liu et al., "Wireless information and power transfer: A dynamic power splitting approach", IEEE Trans. Commun., vol. 61, vol. 9, pp. 3990-4001, Sep. 2013.

[6] I. Krikidis et al., "A low complexity antenna switching for joint wireless information and energy transfer in MIMO relay channels", IEEE Trans. Commun., vol. 62, vol. 5, pp. 1577-1587, May 2014.

[7] M. Di Renzo and W. Lu, "System-level analysis and optimization of cellular networks with simultaneous wireless information and power transfer: Stochastic geometry modeling", IEEE Trans. Veh. Technol., vol. 66, vol. 3, pp. 2251-2275, Mar. 2017.

[8] W. Lu and M. Di Renzo, "Stochastic geometry modeling of cellular networks: Analysis, simulation and experimental validation", in Proc. ACM MSWiM, Nov. 2015, pp. 179-188.

[9] M. Fréchet, "Sur les tableaux de corrélation dont les marges son donnés", Annales de l'Université de Lyon, Série 3, vol. 4, pp. 53-57, 1951.

[10] T. T. Lam et al., "System-level analysis of receiver diversity in SWIPTenabled cellular networks", IEEE J. Commun. Nets., vol. 18, vol. 6, pp. 926-937, Dec. 2016 